\title{
EEG spectral analysis of attention in ADHD: implications for neurofeedback training?
}

\author{
Hartmut Heinrich ${ }^{1,2}{ }^{*}$, Katrin Busch ${ }^{1}$, Petra Studer ${ }^{1}$, Karlheinz Erbe ${ }^{3}$, Gunther H. Moll ${ }^{1}$ and Oliver Kratz ${ }^{1}$ \\ 1 Department of Child and Adolescent Mental Health, University Hospital of Erlangen, Erlangen, Germany \\ ${ }^{2}$ Heckscher-Klinikum, München, Germany \\ ${ }^{3}$ Practice of Child and Adolescent Psychiatry, Bamberg, Germany
}

Edited by:

Tomas Ros, University of Geneva,

Switzerland

Reviewed by:

Juliana Yordanova, Institute of

Neurobiology, Bulgarian Academy

of Sciences, Bulgaria

Tonia A. Rihs, University of Geneva,

Switzerland

*Correspondence:

Hartmut Heinrich, Department of

Child and Adolescent Mental

Health, University Hospital of

Erlangen, Schwabachanlage 6+10,

D-91054 Erlangen, Germany

e-mail:hartmut.heinrich@

uk-erlangen.de
Objective: In children with attention-deficit/hyperactivity disorder (ADHD), an increased theta/beta ratio in the resting EEG typically serves as a rationale to conduct theta/beta neurofeedback (NF) training. However, this finding is increasingly challenged. As NF may rather target an active than a passive state, we studied the EEG in a condition that requires attention.

Methods: In children with ADHD of the DSM-IV combined type (ADHD-C; $N=15$ ) and of the predominantly inattentive type (ADHD-I; $N=9$ ) and in typically developing children $(N=19)$, EEG spectral analysis was conducted for segments during the attention network test (ANT) without processing of stimuli and overt behavior. Frontal (F3, Fz, F4), central $(\mathrm{C} 3, \mathrm{Cz}, \mathrm{C} 4)$ and parietal (P3, Pz, P4) electrodes were included in the statistical analysis. To investigate if EEG spectral parameters are related to performance measures, correlation coefficients were calculated.

Results: Particularly in the ADHD-C group, higher theta and alpha activity was found with the most prominent effect in the upper-theta/lower-alpha $(5.5-10.5 \mathrm{~Hz})$ range. In the ADHD-I group, a significantly higher theta/beta ratio was observed at single electrodes (F3, Fz) and a tendency for a higher theta/beta ratio when considering all electrodes (large effect size). Higher $5.5-10.5 \mathrm{~Hz}$ activity was associated with higher reaction time variability with the effect most prominent in the ADHD-C group. A higher theta/beta ratio was associated with higher reaction times, particularly in the ADHD-I group.

Conclusions: (1) In an attention demanding period, children with ADHD are characterized by an underactivated state in the EEG with subtype-specific differences. (2) The functional relevance of related EEG parameters is indicated by associations with performance (reaction time) measures. (3) Findings provide a rationale for applying NF protocols targeting theta (and alpha) activity and the theta/beta ratio in subgroups of children with ADHD.

Keywords: neurofeedback, ADHD, EEG, spectral analysis, attention, brain-behavior-relationship, subtypes

\section{INTRODUCTION}

Theta/beta training belongs to the neurofeedback (NF) protocols which are frequently applied in children with ADHD; for review see Arns et al. (2014) and Gevensleben et al. (2014). In theta/beta training, the aim is to decrease theta activity and to increase activity in the beta band of the EEG or to decrease the theta/beta ratio with feedback being calculated typically from electrode Cz. In randomized controlled trials, it has been found to be superior in reducing the children's inattentive, hyperactive and impulsive behavior (medium effect sizes) compared to computerized attention training (Gevensleben et al., 2009a) and EMG biofeedback (Bakhshayesh et al., 2011).

Specificity of training effects is further supported by findings at the neurophysiological level (Gevensleben et al., 2009b). Higher baseline theta activity in the resting EEG (recorded in an eyes open condition) over centro-parietal regions was associated with a larger reduction of the severity of ADHD symptoms after theta/beta training and larger decreases of theta activity from preto post-training were accompanied by larger clinical improvements. These findings also indicate that it should be possible to derive EEG-based indication criteria for which children theta/beta training may be more appropriate.

As a rationale for applying theta/beta training in ADHD, authors typically referred to findings from resting EEG studies comparing children with ADHD to typically developing controls (see e.g., Heinrich et al., 2007).

\section{RESTING EEG STUDIES IN ADHD}

A series of resting EEG studies in ADHD (eyes open and eyes closed condition) have been conducted since the 1980s and 
reviewed e.g., in Barry et al. (2003). Consistently, elevated levels of theta activity and reduced relative levels of beta and alpha activity (corresponding to increased theta/beta and theta/alpha ratios) were found compared to typically developing children. Slow activity was described to have a fronto-central distribution although group differences were most prominent over posterior regions (Banaschewski and Brandeis, 2007). Deviances appeared to be more prominent in the DSM-IV combined type of ADHD compared to the predominantly inattentive subtype. ${ }^{1}$

The theta/beta ratio measured at $\mathrm{Cz}$ was reported to discriminate reliably between children with ADHD and controls (classification rate: ca. 90\%; Monastra et al., 1999; Snyder et al., 2008). On the other hand, Barry et al. (2003) stated EEG heterogeneity in ADHD and suggested to define EEG-based subtypes of ADHD.

Applying theta/beta training was thought to "normalize" the cortical slowing. However, recent studies question if the major part of children with ADHD are actually characterized by an increased theta/beta ratio in the resting EEG. Arns et al. (2013) conducted a meta-analysis studying theta/beta ratio in an eyesopen condition at electrode $\mathrm{Cz}$. Including nine studies with about 1200 children and adolescents with ADHD and about 500 children without ADHD, they found a medium effect size of 0.62 (age range from 6 to 18 years). However, the authors argued that this number is misleading as post hoc analysis revealed a decreasing difference in theta/beta ratio across years due to an increasing theta/beta ratio for the non-ADHD (control) participants.

This point of view is further supported by two studies, which were published after this meta-analysis and did not find differences between children with ADHD and typically developing children in any frequency band considered (Liechti et al., 2013; Buyck and Wiersema, 2014). However, subdividing the ADHD group revealed increased theta/beta ratios in children and adults of the predominantly inattentive subtype in Buyck and Wiersema (2014) who analyzed EEG activity at midline electrodes.

\section{EEG STUDIES IN ADHD DURING TASK PERFORMANCE}

Interpreting NF as a neurobehavioral approach, training rather targets an active than a passive state (Gevensleben et al., submitted). For example, training may also comprise trials combined with tasks (e.g., reading, listening). In this respect, it appears to be more relevant to consider the EEG during task processing though it has to be kept in mind that the resting EEG does not only reflect a trait but also a state marker (Hagemann et al., 2005).

Up to now, EEG profiles in ADHD during cognitive tasks have less often been studied. Monastra et al. (1999) did not only study the theta/beta ratio in a resting condition at single electrode $\mathrm{Cz}$ but also while children were reading, listening and drawing. For all conditions, the ADHD group was characterized by increased theta/beta ratios. In El-Sayed et al. (2002), increased slow activity (mainly over frontal electrodes) was found especially during an

\footnotetext{
${ }^{1}$ In the fourth edition of the Diagnostic and Statistical Manual of Mental Disorders (DSM-IV; American Psychiatric Association, 2000), three subtypes of ADHD (combined type, predominantly inattentive type, predominantly hyperactive-impulsive type) are distinguished reflecting the heterogeneity of the disorder. In clinical practice, the combined type appears to be most common. Besides the combined type, research focuses also on the predominantly inattentive type.
}

attention (continuous performance) task but also during eyesopen resting condition.

Loo and Smalley (2008) investigated familiality of spectral EEG measures in ADHD during resting and cognitive activation (sustained attention task) conditions. Effects were clearly stronger for the activation compared to the resting conditions and did not show topographic specificity. Sibling correlations of 0.6-0.7 were obtained for the theta, alpha and beta band. Theta and alpha power were associated with task performance (reaction time variabililty, omission errors). So, not only theta and beta activity but also alpha activity should be considered when studying EEG activity during an activation condition in the context of ADHD. However, in our opinion, two points were not realized in an optimal way in the study of Loo and Smalley (2008). First, associations between EEG and performance measures were not controlled for potential developmental effects and, second, we would prefer to analyze EEG segments reflecting an attentive state without processing of task-related information (stimuli) as eventrelated EEG components interfere with the spontaneous activity.

Lazzaro et al. (2001) reported increased pre-stimulus theta activity in children with ADHD during an oddball task. Theta activity correlated inter alia with the latency of the event-related potential component P3 indexing attention.

\section{INFORMATION ABOUT THE DATASET/OBJECTIVES OF THE STUDY}

In the present case-control study, we conducted EEG spectral analysis in children with ADHD during an attentive state. For this analysis, we used a previously published dataset (Kratz et al., 2011). In Kratz et al. (2011), attentional processing was studied in children with ADHD during the attention network test (ANT). At the neural level (event-related potentials), deviant cue processing (reduced cue-P3) was the most prominent effect. The contingent negative variation $(\mathrm{CNV})^{2}$ reflecting inter alia cognitive preparation processes was not found to be smaller-probably due to the younger age of this sample compared to other studies (e.g., Albrecht et al., 2013). Differences between ADHD subtypes (combined type vs. predominantly inattentive type) could be observed.

Using this dataset for the present analysis allowed to consider segments during the ANT reflecting a state of activation/(tonic) alertness and free of stimulus processing. We expected that children with ADHD show increased theta activity and/or an increase theta/beta ratio across the scalp surface during an attentive state serving as a rationale to apply related protocols in NF training.

We were also interested in comparing DSM-IV subtypes of ADHD. In order to learn more about the functional significance of the spectral EEG parameters, we studied associations (correlations) between these spectral EEG parameters and performance measures (particularly reaction time measures).

\section{MATERIALS AND METHODS PARTICIPANTS}

Fifteen children with ADHD of the combined type (ADHD-C; according to DSM-IV criteria), nine children with ADHD of the predominantly inattentive subtype (ADHD-I) and 19 typically

\footnotetext{
${ }^{2} \mathrm{~A}$ reduced $\mathrm{CNV}$ is typically considered as a rationale to apply training of slow cortical potentials (SCPs) in children with ADHD (Heinrich et al., 2007).
} 
developing children were included in the study. Children had to be aged $8-11$ years and to have a full-scale IQ of at least 80 . All children had normal or corrected-to-normal vision. Adequate task performance in the ANT and sufficient EEG data quality was also necessary to be included in this study (for details see below). The three groups (ADHD-C, ADHD-I, controls) were comparable regarding age and sex (Demographic and clinical variables of the sample are summarized in Table 1). IQ was significantly lower in the ADHD-I group but IQ had no significant influence on the group-specific results as tested by comparing the ADHD-I group to a subgroup of typically developing children with comparable IQ; see also Kratz et al. (2011).

Patients were either recruited from a child and adolescent psychiatric practice in Bamberg (Germany) and took part in a medication trial (Kratz et al., 2012) or were recruited via the outpatient department of the Department of Child and Adolescent Mental Health at the University Hospital of Erlangen and participated in a NF trial (Gevensleben et al., 2009a). Baseline measurements (conducted before starting treatment) were considered for the present analysis. Diagnostics comprised a clinical interview conducted by a child and adolescent psychiatrist or a clinical psychologist. ADHD diagnoses were confirmed using the Diagnostic Checklist for Hyperkinetic Disorders/ADHD (Döpfner and Lehmkuhl, 2000). Patients had no comorbid diagnoses other than oppositional defiant disorder, emotional disorder and dyslexia. All children with ADHD included in this study were drug-naive. Typically developing children were recruited from the personal environment of employees of the clinic. For none of the children of the control group, parents reported a psychiatric or neurological disorder.

For all children, the German ADHD rating scale (FBB-HKS: Döpfner and Lehmkuhl, 2000) was assessed (filled out by parents). The FBB-HKS is a 20 -item questionnaire related to the DSM-IV and ICD-10 criteria for ADHD (nine inattention items, seven hyperactivity items, four impulsivity items). Severity of each item is rated on a scale from 0 to 3 . The questionnaire provides a total score (mean value of all 20 items) as well as subscores for inattention and hyperactivity/impulsivity. For the typically developing children included in the study, FBB-HKS scores (total score and subscales) were not more than one standard deviation above normative means. Control and ADHD groups differed on all FBB-HKS scales $\left(F_{(2,40)}>46.9 ; p<0.001\right)$. For the two ADHD groups (ADHD-C vs. ADHD-I), the FBB-HKS total score $\left(t_{(22)}=1.38\right.$, n.s. $)$ and the score for the inattention subscale $\left(t_{(22)}=-0.20\right.$, n.s. $)$ were comparable. However, the score for the hyperactivity/impulsivity subscale was higher in the ADHD-C group $\left(t_{(21)}=2.45 ; p<0.05\right)$.

The study, which was approved by the Ethics Committee of the Medical Faculty of the University of Erlangen-Nuremberg, was conducted in accordance with the Declaration of Helsinki. Children gave their assent and parents provided written informed consent.

\section{PROCEDURE AND TASK}

In the testing session, children sat on a comfortable chair in front of a computer monitor (viewing distance: $72 \mathrm{~cm}$ ). During EEG preparation, the children could watch age-appropriate films. The ANT, which consisted of four blocks of 48 trials each, lasted about 15 min (including short breaks between the task blocks). During the test brain electrical activity was recorded. The children received standardized instructions before performing a practice block of 24 trials. After each task block, a summary of the task performance was shown on the screen.

Children were instructed to "feed" a hungry fish that would appear above or below a fixation cross. If the fish pointed to the right (resp. left) side, the children had to press the right (resp. left) mouse button in order to feed the fish. This target fish was the center fish in a row of five fish with the flanking fish either looking in the same direction (congruent condition) or in the opposite direction (incongruent condition).

One of three cue conditions (equal probability) preceded the presentation of the fish: in the NeutralCue condition, an asterisk

Table 1 | Sample characteristics and performance data of the attention network test.

\begin{tabular}{|c|c|c|c|c|}
\hline & \multicolumn{2}{|c|}{ Children with ADHD } & Controls $(N=19)$ & Statistics \\
\hline Age (months) & $117.8 \pm 12.0$ & $112.6 \pm 12.6$ & $122.0 \pm 11.9$ & $F_{(2,40)}=1.9$, n.s. \\
\hline $\operatorname{Sex}(m / f)$ & $10 / 5$ & $8 / 1$ & $15 / 4$ & $x^{2}=1.65$, n.s. \\
\hline \multicolumn{5}{|c|}{ German ADHD rating scale (FBB-HKS) } \\
\hline Total score & $1.56 \pm 0.37$ & $1.34 \pm 0.38$ & $0.34 \pm 0.22$ & $F_{(2,40)}=67.9, p<0.001$ \\
\hline \multicolumn{5}{|l|}{ Associated disorders } \\
\hline Oppositional defiant disorder & 2 & 0 & - & \\
\hline Emotional disorder & 1 & 1 & - & \\
\hline Dyslexia & 1 & 2 & - & \\
\hline \multicolumn{5}{|l|}{ Attention network test } \\
\hline Hits (correct responses) & $171.5 \pm 19.4$ & $181.0 \pm 5.8$ & $175.1 \pm 17.2$ & $F_{(2,40)}=0.9$, n.s. \\
\hline
\end{tabular}


at the center of the screen indicated that the target fish was about to appear soon. In the SpatialCue condition, an asterisk was presented at the location of the target fish, indicating not only that the target was about to appear soon but also its location on the screen. In the NoCue condition, the fish were presented without a cue stimulus.

A schematic illustration of the ANT as applied in the present study, including technical details is presented in Figure 1. The test was realized in Presentation (Neurobehavioral Systems, Albany, CA, USA).

\section{EEG RECORDING AND PREPROCESSING}

A Brainamp recording system (Brainamp standard amplifier, Brain Products, Munich, Germany) was used. Brain electrical activity was recorded from 23 sintered $\mathrm{Ag} / \mathrm{AgCl}$ electrodes (10/20 system; Fpz, Oz, mastoids). Positions for reference and ground electrode were $\mathrm{FCz}$ and $\mathrm{CPz}$, respectively. Vertical and horizontal electrooculogram was recorded from electrodes placed above and below the right eye and at the outer canthi. A sampling rate of $500 \mathrm{~Hz}$ was used. Filter bandwidth at recording was $0.016-120 \mathrm{~Hz}$. Impedances were kept below $20 \mathrm{k} \Omega$.

For preprocessing and data analysis, the VisionAnalyzer software (Brain Products, Gilching, Germany) was used. After applying a $50 \mathrm{~Hz}$ notch filter and bandpass filtering $(0.05-30 \mathrm{~Hz}$,
$24 \mathrm{~dB} /$ oct Butterworth filters), eye movement artifacts were corrected using independent component analysis (ICA, Jung et al., 2000). Signals were re-referenced to linked-mastoids. If amplitudes exceeded $\pm 100 \mu \mathrm{V}$ at any electrode, a segment of -300 to $+700 \mathrm{~ms}$ around this artifact was excluded from further analyses.

EEG spectral analysis was conducted for NoCue segments of $1.5 \mathrm{~s}$ length, i.e., for segments before the onset of flanker stimuli which were not preceded by a cue stimulus. These were the segments with the longest "pure" EEG period without processing of cue or target stimuli and correspond to an attentive state.

For each child, at least 20 artefact-free segments (followed by a correct response to the target stimulus) had to be available. The number of segments without artefacts were slightly but not significantly smaller in the ADHD groups (control: $47.2 \pm 9.6$; ADHD-C: $44.3 \pm$ 9.8; ADHD-I: $38.1 \pm 14.6 ; F_{(2,40)}=2.13$, $p=0.13)$.

\section{DATA ANALYSIS}

The number of hits, median of reaction times and reaction time variability were determined. ANT-specific performance measures (alerting score, orienting score and conflict score; Fan et al., 2002) had not been significantly different for ADHD groups and control group in Kratz et al. (2011). So, for simplicity, they will be omitted in this manuscript. Reaction time measures were based on trials

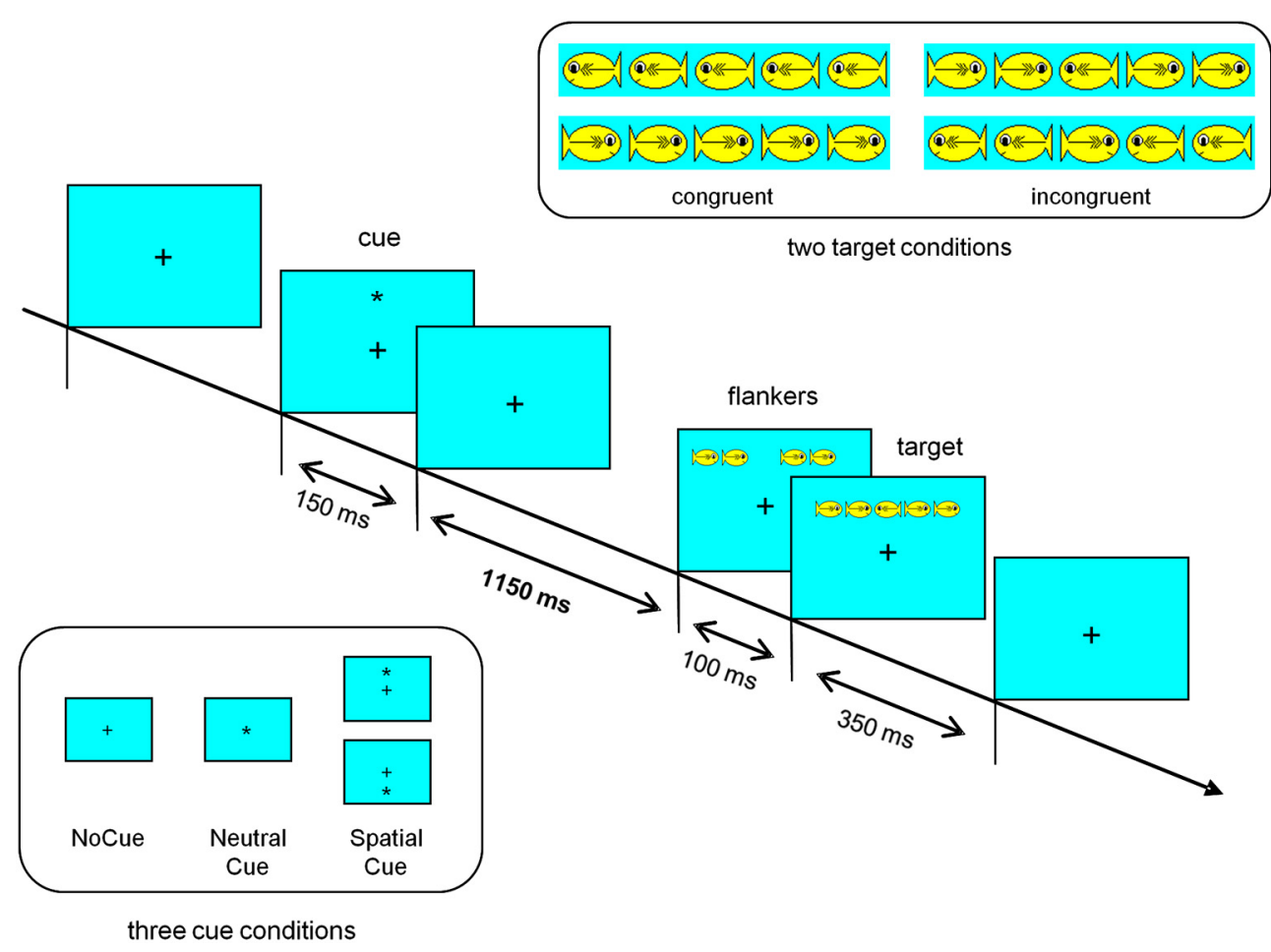

FIGURE 1 | Schematic illustration of the attention network test (ANT) as applied in our study. A fixation cross located on the center of the computer screen was shown during the complete test. The row of hungry fish appeared above or below the fixation cross (about $1^{\circ}$ ). The target fish, i.e., the center fish, was visible for $350 \mathrm{~ms}$. Presentation of the flanking fish started $100 \mathrm{~ms}$ before the center fish appeared. Cue stimuli were visible for $150 \mathrm{~ms}$, starting $1400 \mathrm{~ms}$ before the target stimulus (fish). A cue stimuli was always followed by a target stimulus. In comparison to the original child version of the ANT (Rueda et al., 2004), a longer interval between cue and target stimulus was used $(1400 \mathrm{~ms}$ instead of $600 \mathrm{~ms}$ ) to elicit a contingent negative variation. Each fish subtended $1.6^{\circ}$ of visual angle and the contours of adjacent fish were separated by $0.21^{\circ}$. The intertrial interval varied randomly between 3.5 and $5.0 \mathrm{~s}$. 
with correct responses. Only trials with reaction times between 200 and $1500 \mathrm{~ms}$ after target stimulus onset were included in the analysis.

For each NoCue trial, a voltage density spectrum was computed after applying a Hanning window and these spectra were averaged then. From the averaged spectra, voltage values for theta $(4-7.5 \mathrm{~Hz})$, alpha $(7.5-12.5 \mathrm{~Hz})$, beta $(12.5-20 \mathrm{~Hz})$ band as well as the theta/beta ratio were calculated at different electrodes (F3, Fz, F4, C3, Cz, C4, P3, Pz, P4). In Figure 2, the grand average spectra for control, $\mathrm{ADHD}-\mathrm{C}$ and $\mathrm{ADHD}-\mathrm{I}$ groups are depicted. Based on visual inspection, the largest differences between the groups seem to occur within an upper-theta/lower-alpha (5.5-10.5 Hz) band. Therefore, we decided to consider this band in addition to the traditional EEG bands.

\section{STATISTICAL ANALYSIS}

Performance data (hits, reaction time measures) were analyzed using a one-way ANOVA with between-subject factor GROUP (control, ADHD-C, ADHD-I). $t$-tests were applied for post hoc analysis (pairwise comparisons of two groups) using BonferroniHolm correction to control for multiple comparisons.
For the different EEG frequency bands, repeated-measure ANOVAs were computed with between-subject factor GROUP and repeated-measurement (electrode) factors $\mathrm{Y}$ (frontal $[\mathrm{F} 3, \mathrm{Fz}$, F4], central [C3, Cz, C4], parietal $[\mathrm{P} 3, \mathrm{Pz}, \mathrm{P} 4])$ and $\mathrm{X}$ (left [F3, $\mathrm{C} 3, \mathrm{P} 3]$, midline $[\mathrm{Fz}, \mathrm{Cz}, \mathrm{Pz}]$, right $[\mathrm{F} 4, \mathrm{C} 4, \mathrm{P} 4])$ to test potential topography/laterality effects. Post hoc analysis also comprised correction for multiple comparisons (Bonferroni-Holm). Corrected $p$-values are reported.

Associations between EEG spectral parameters and performance measures were studied focusing on those EEG measures for which largest group-specific effects were obtained in the before-mentioned analysis. We controlled for age-related effects. However, as the portion of 8 year-old children was higher in the ADHD groups, controlling/correcting for agerelated changes by considering the complete sample would lead to an overestimation of age-related changes at the cost of grouprelated effects. Instead, we decided to correct for age-related changes in the complete sample based on the regression coefficient of the control group. Pearson's correlation coefficients were calculated for potentially age-corrected measures. If significant correlations were found for the complete sample,

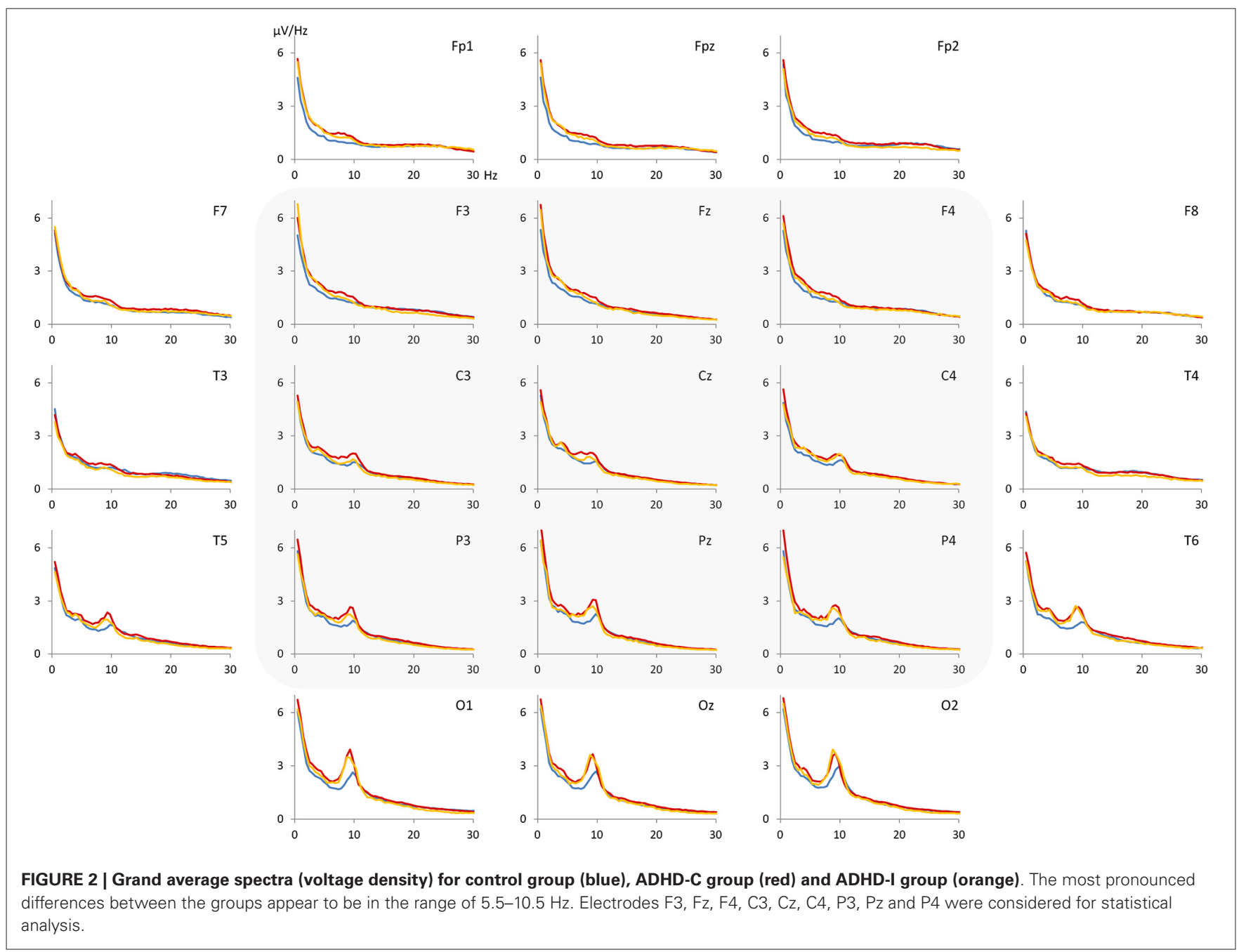


Table 2 | EEG measures and statistical results

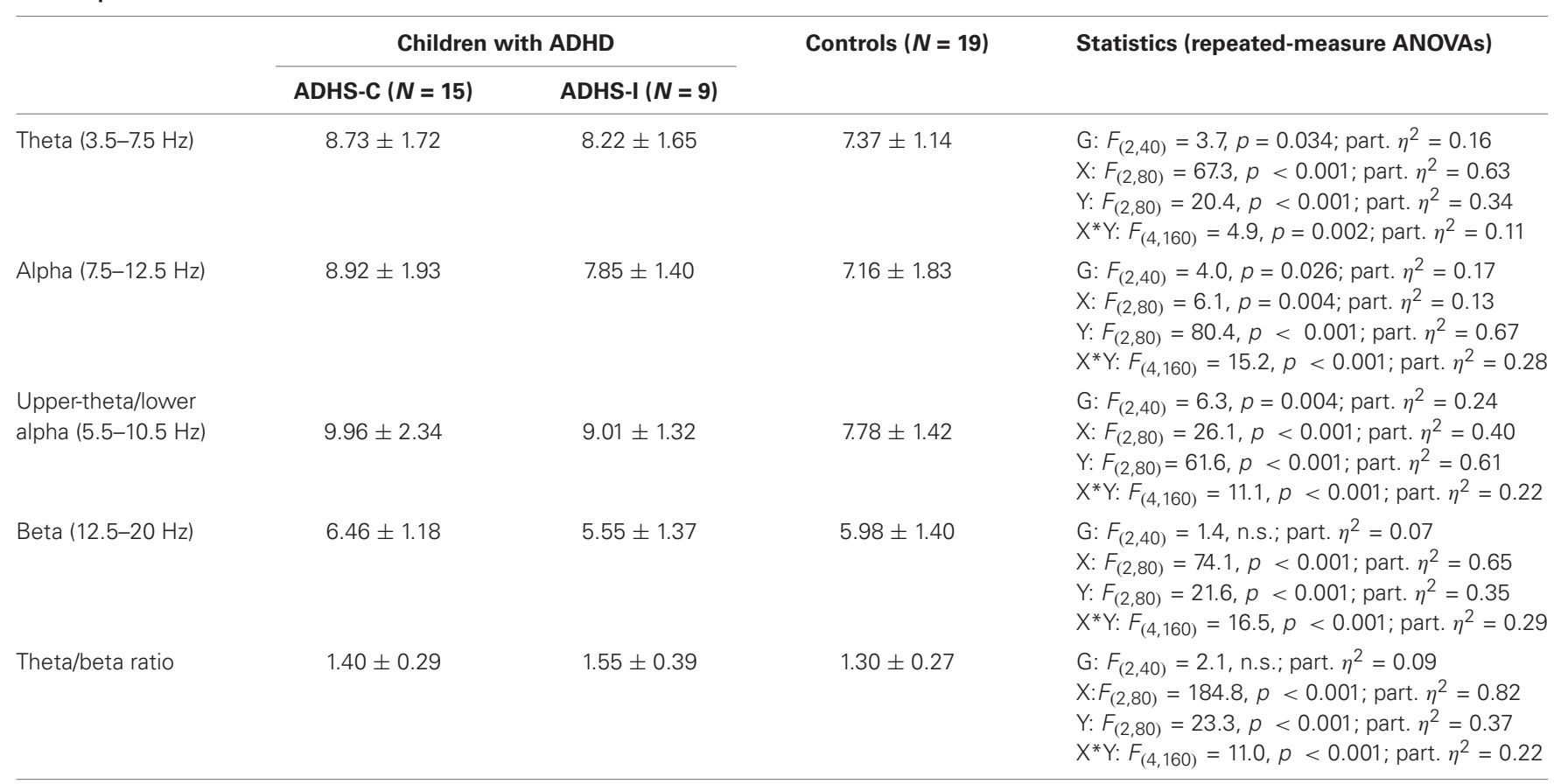

For control and ADHD groups, the group's mean (for the average regarding the different EEG frequency bands over frontal, central and parietal electrodes considered in the ANOVAs) $\pm S D$ is presented. Unit (except theta/beta ratio): $\mu \mathrm{V}$. For the repeated-measure ANOVAs, the results obtained for the between-subject factor Group $(G)$, the within-subjects factors $X$ (left, midline, right) and $Y$ (frontal, central, parietal) and their interaction are provided.

we also tested ADHD groups separately to exclude spurious correlations.

IBM SPSS Statistics (Version 20.0) was used for statistical analysis.

\section{RESULTS \\ PERFORMANCE MEASURES}

Results of performance measures are summarized in Table $\mathbf{1 .}$ Reaction time variability was significantly higher in the two ADHD groups compared to the control group (control vs. ADHD-I: $t_{(26)}=-3.32 ; p$ (corr.) $=0.009$; control vs. ADHD-C: $t_{(32)}=-2.61 ; p$ (corr. $\left.)=0.03\right)$. For the median of reaction times, a GROUP effect was obtained due to higher reaction times in the ADHD-I group in comparison to the control group (control vs. ADHS-I: $t_{(26)}=-3.71 ; p($ corr. $\left.)=0.003\right)$ as well as the ADHD-C group (ADHD-C vs. ADHD-I: $t_{(22)}=-2.36 ; p($ corr. $\left.)=0.05\right)$.

\section{SPECTRAL EEG PARAMETERS}

Results of the ANOVAs for the different frequency bands are summarized in Table 2. For all frequency bands considered, the repeated-measure ANOVAs revealed (highly) significant effects for the within-subject factors $\mathrm{X}, \mathrm{Y}$ and their interaction $\mathrm{X}^{*} \mathrm{Y}$ related to the topography of the EEG activity in the different frequency bands. Theta, alpha and $5.5-10.5 \mathrm{~Hz}$ activity were highest at electrode $\mathrm{Pz}$ (parietal, midline); see also Figure 3. The highest beta activity was measured at left and right frontal electrodes (F3 and F4). The theta/beta ratio had its maximum at electrode $\mathrm{Cz}$. No significant interaction effect containing the factor Group was obtained, i.e., topography did not differ significantly between the groups.

For theta activity, alpha activity and particularly uppertheta/lower-alpha $(5.5-10.5 \mathrm{~Hz})$ activity, significant group main effects indicated higher activity in the ADHD groups. Post hoc analysis revealed that $5.5-10.5 \mathrm{~Hz}$ activity was higher particularly in the ADHD-C group (control vs. ADHD-C: $t_{(32)}=-3.35$; $p$ (corr. $)=0.006$ ) and to a smaller extent in the ADHD-I extent in the ADHD-I group (control vs. ADHD-I: $t_{(26)}=-2.17$; $p$ (corr. $)=0.08, p$ (uncorr. $)=0.04)$.

No significant main effect for the theta/beta ratio was found. However, a medium effect size (part. $\eta^{2}=0.09$ ) may indicate some effect which did not turn out to be significant due to the limited sample size. So, we decided to look at the theta/beta ratio in more detail in an exploratory way. At least a tendency for a higher theta/beta ratio (averaged over the nine electrodes) in the ADHDI group (control vs. ADHD-I: $t_{(26)}=-2.0 ; p=0.057$; Cohen's $d=0.8$ ) was obtained whereas no effects were observed for the ADHD-C group (control vs. ADHD-C: $t_{(32)}=-1.02$; n.s.). When group at single electrodes, significant effects were found for electrodes $\mathrm{F} 3$ and $\mathrm{Fz}$ (control vs. ADHD-I: $t_{(26)} \leq-2.28 ; p \leq 0.03$ ).

\section{ASSOCIATIONS BETWEEN SPECTRAL EEG PARAMETERS AND PERFORMANCE MEASURES}

As only GROUP main effects were found in the ANOVAs, we considered the average of all electrodes for the correlational analysis. A significant correlation was found between the activity in the $5.5-10.5 \mathrm{~Hz}$ band (averaged over frontal, central and parietal electrodes) and reaction time variability 


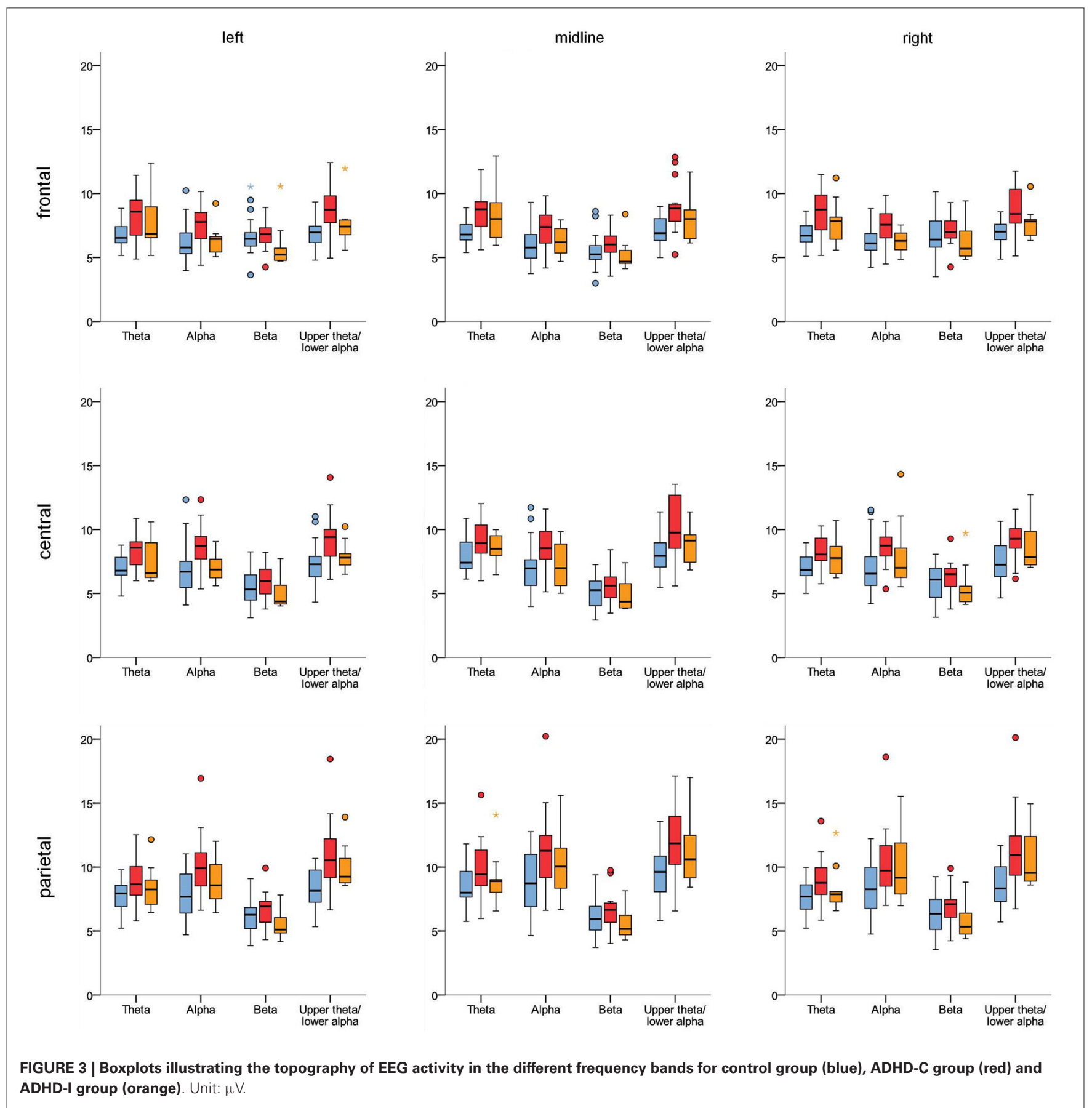

$(r=0.34, p=0.025)$ : Higher activity in the $5.5-10.5 \mathrm{~Hz}$ band was associated with higher reaction time variability (see Figure 4A). For the ADHD-C group, the correlation coefficient was 0.48 .

A significant correlation was also obtained between the theta/beta ratio (averaged over frontal, central and parietal leads) and the median of reaction times $(r=0.42, p=0.005)$; see Figure 4B. The higher the theta/beta ratio was, the longer reaction times were. This effect was most prominent in the ADHD-I group $(r=0.54)$.
Hence, significant associations were found for those frequency bands and performance (reaction time) measures with deviations in the ADHD groups. It has to be noted that these correlations did not reach statistical significance in the respective ADHD group due to the small group sizes.

\section{DISCUSSION}

In the present study, we conducted EEG spectral analysis during an attention demanding period in children with ADHD (compared to typically developing controls). Deviant EEG patterns 


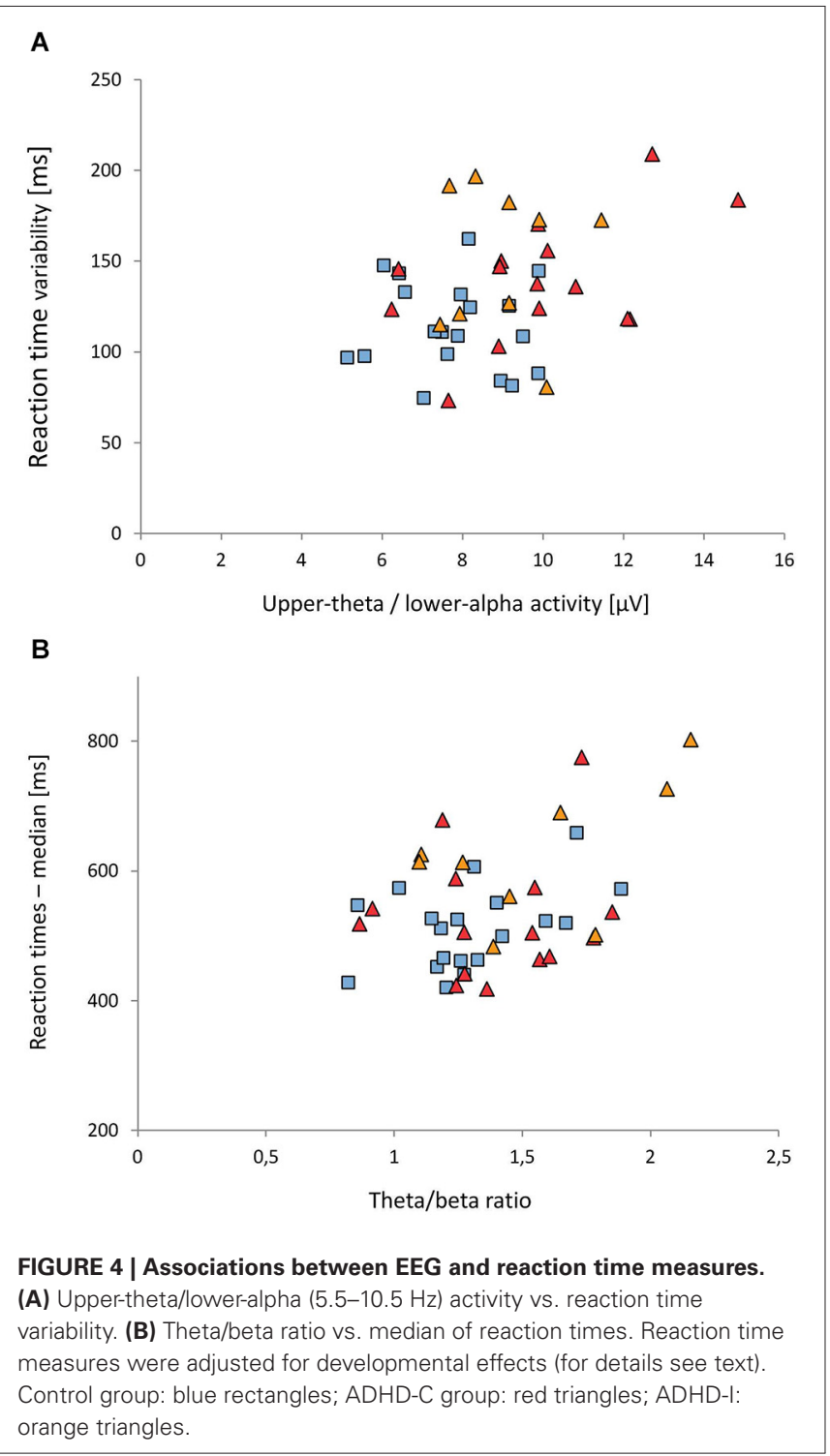

were obtained with subtype-specific differences between the DSM-IV combined type and the predominantly inattentive subtype.

\section{SPECTRAL EEG MEASURES DURING AN ATTENTIVE STATE IN ADHD (SUBTYPES)}

In contrast to recent resting-EEG studies (e.g., Ogrim et al., 2012; Liechti et al., 2013), significant differences related to the theta band and the alpha band were obtained between children with ADHD and typically developing children: activity in these frequency band was significantly larger in children with ADHD. In the $\mathrm{ADHD}-\mathrm{C}$ group, effects were most prominent when considering the $5.5-10.5 \mathrm{~Hz}$ (upper-theta/lower-alpha) band. Global statistical analysis did not reveal a significant group effect for the theta/beta ratio, i.e., the major part of the children with ADHD was not characterized by an increased theta/beta ratio. On the other hand, a large effect size for the comparison of control and ADHD-I group may indicate an increased theta/beta ratio in children of the predominantly inattentive subtype comparable to the findings of Buyck and Wiersema (2014) obtained in the resting EEG. However, this finding is limited by the rather small size of our ADHD-I group.

As inattention scores of the German ADHD rating scale were comparable for ADHD-C group and ADHD-I group we argue that the differential pattern does not reflect different severity of inattention symptoms but rather suggest that there are different neural mechanisms accounting for attentional dysfunctions in ADHD subtypes. In Heinrich et al. (submitted), we had already reported different distributions of cue-P3 single trial amplitudes for the two ADHD groups further strengthening this point of view.

\section{ASSOCIATIONS BETWEEN SPECTRAL EEG PARAMETERS AND REACTION TIME MEASURES}

Interestingly, significant (positive) correlations between those spectral EEG parameters and reaction time measures $(5.5-10.5 \mathrm{~Hz}$ activity and reaction time variability; theta/beta ratio and median of reaction times) were found for which differences between the ADHD groups and the control group had been obtained. These associations suggest a functional relevance of the EEG parameters, particularly in the context of ADHD: a suboptimal neural state at stimulus presentation results in impaired performance. As we controlled for age effects and also considered the ADHD groups separately, it seems rather unlikely that the correlations obtained for our data reflect spurious correlations.

Loo and Smalley (2008) had also reported a positive correlation between reaction time variability and activity in the theta and alpha band during an attention (continuous performance) test. Increased reaction time variability is a robust finding in children with ADHD with medium to large effect sizes being reported (meta-analysis for example in Kofler et al., 2013: Hedges' $g=0.76$ ). Increased activity in the upper-theta/loweralpha band, which may be interpreted as an underactivated neural state, could reflect a neural mechanism underlying increased reaction time variability in ADHD besides top-down control and motor preparation processes (Karalunas et al., 2014). It seems unlikely that slower reaction times in the ADHD-I group of our sample are mainly due to very slow reaction times in a few trials but they rather reflect a generally slower processing/response style. Findings indicate that this slowing may be related to a higher theta/beta ratio. The differential associations regarding ADHD-C and ADHD-I groups further support the notion of distinct neural mechanisms underlying attentional dysfunctions in ADHD subtypes.

\section{POTENTIAL IMPLICATIONS FOR NEUROFEEDBACK TRAINING IN ADHD}

NF may be interpreted as an approach to gain self-control over a certain aspect of neural activity associated with a specific cognitive or emotional state (Gevensleben et al., submitted). In this respect, findings of the present study may have the following implications for NF training in ADHD.

In children with ADHD of the combined type, an uppertheta/lower-alpha $(5.5-10.5 \mathrm{~Hz})$ protocol associated with an 
attentive state may be more effective than theta/beta training. It will have to be studied if indication criteria for the use of a specific protocol based on (inter alia) EEG characteristics at pre-training can be developed. As theta activity in the resting EEG at pretraining was found to be a predictor for the effects of theta/beta training (Gevensleben et al., 2009b), this seems to be a realistic task.

Up to now, only a single EEG channel is typically used to calculate feedback information in EEG NF training. For theta/beta training in $\mathrm{ADHD}$, most often electrode $\mathrm{Cz}$ is considered. In our data, increased upper-theta/lower-alpha activity in the ADHD$\mathrm{C}$ group and a higher theta/beta ratio in the ADHD-I group were not topographically specific, i.e., they were not restricted to/particularly pronounced at a certain electrode. Looking at single electrodes, effects at electrode $\mathrm{Cz}$ appeared rather smaller than larger compared to frontal, electrodes (F3, Fz).

It has to be taken into consideration that frontal midline theta (associated with working memory and cognitive control processes; Jensen and Tesche, 2002; Enriquez-Geppert et al., 2014) could interfere with the more generalized theta pattern addressed for example in theta/beta training if feedback information is calculated from $\mathrm{Cz}$ only. So, in our opinion, a more robust/more specific feedback signal may be obtained if not a single channel but a combination of several electrodes is used. If NF training does not target a topographically specific EEG pattern, the average of a grid of distributed electrodes may be preferable.

NF training trials may also be combined with attention tasks to facilitate training effects at the performance level: depending on the protocol applied, faster or less variable reaction times may be achieved. Regarding other tasks (e.g., reading, listening), it will have to be tested whether refined frequency bands and feedback parameters, respectively, may also be more characteristic for children with ADHD.

\section{LIMITATIONS OF OUR STUDY}

Findings are limited by the generally small sample size. However, we'd see sample size more critical if findings had not turned out to be significant. Large effect sizes were obtained and effects were not just due to outliers suggesting clear differences in the distribution of control and ADHD groups. In any case, larger samples will have to be studied to see if results are confirmed and to what extend EEG-based subtypes can be found.

We could not compare resting and active EEG conditions directly. Thus, it cannot be excluded that corresponding effect sizes could have also been found in the resting EEG of our sample. However, in our opinion, our findings complement/are compatible with results of recent studies that either report no significant global differences in recent resting EEG studies (e.g., Liechti et al., 2013; Buyck and Wiersema, 2014) and/or more pronounced effects in active compared to resting conditions (e.g., Loo and Smalley, 2008).

\section{CONCLUSIONS}

During an attentive state, children with ADHD are characterized by an underactivated state in the EEG with subtype-specific differences. Whereas the most prominent effect was obtained for the upper-theta/lower alpha $(5.5-10.5 \mathrm{~Hz})$ range in children of the combined type, hints for an increased theta/beta ratio were found in children of the predominantly inattentive subtype. The functional relevance of these EEG parameters was indicated by associations with reaction time measures, which were pronounced most in the ADHD groups. Findings may provide a rationale for applying NF training protocols targeting theta activity and theta/beta ratio in subgroups of children with ADHD to achieve an attentive state. In this respect, it will be interesting if indication criteria for a specific protocol in an individual child can be developed which can be applied in clinical practice.

\section{ACKNOWLEDGMENTS}

We acknowledge support by Deutsche Forschungsgemeinschaft and Friedrich-Alexander-Universität Erlangen-Nürnberg (FAU) within the funding programme Open Access Publishing.

We would like to thank Martin Deinzer, Jeska Baack and Susanne Malcherek for their valuable support. We also thank all participating families for their contribution and effort.

\section{REFERENCES}

Albrecht, B., Brandeis, D., Uebel, H., Valko, L., Heinrich, H., Drechsler, R., et al. (2013). Familiality of neural preparation and response control in childhood attention deficit-hyperactivity disorder. Psychol. Med. 43, 1997-2011. doi: 10. 1017/S003329171200270X

American Psychiatric Association. (2000). Diagnostic and Statistical Manual of Mental Disorders. Washington, DC: American Psychiatric Press.

Arns, M., Conners, C. K., and Kraemer, H. C. (2013). A decade of EEG theta/beta ratio research in ADHD: a meta-analysis. J. Atten. Disord. 17, 374-383. doi: 10. 1177/1087054712460087

Arns, M., Heinrich, H., and Strehl, U. (2014). Evaluation of neurofeedback in ADHD: the long and winding road. Biol. Psychol. 95, 108-115. doi: 10.1016/j. biopsycho.2013.11.013

Bakhshayesh, A. R., Hänsch, S., Wyschkon, A., Rezai, M. J., and Esser, G. (2011). Neurofeedback in ADHD: a single-blind randomized controlled trial. Eur. Child Adolesc. Psychiatry 20, 481-491. doi: 10.1007/s00787-011-0208-y

Banaschewski, T., and Brandeis, D. (2007). Annotation: what electrical brain activity tells us about brain function that other techniques cannot tell us-a child psychiatric perspective. J. Child Psychol. Psychiatry 48, 415-435. doi: 10. 1111/j.1469-7610.2006.01681.x

Barry, R. J., Clarke, A. R., and Johnstone, S. J. (2003). A review of electrophysiology in attention-deficit/hyperactivity disorder: I. Qualitative and quantitative electroencephalography. Clin. Neurophysiol. 114, 171-183. doi: 10.1016/s13882457(02)00362-0

Buyck, I., and Wiersema, J. R. (2014). Resting electroencephalogram in attention deficit hyperactivity disorder: developmental course and diagnostic value. Psychiatry Res. 216, 391-397. doi: 10.1016/j.psychres.2013.12.055

Döpfner, M. and Lehmkuhl, G. (2000). DISYPS-KJ-Diagnostik-System für Psychische Störungen im Kindes- und Jugendalter. Bern: Hans Huber.

El-Sayed, E., Larsson, J. O., Persson, H. E., and Rydelius, P. A. (2002). Altered cortical activity in children with attention-deficit/hyperactivity disorder during attentional load task. J. Am. Acad. Child Adolesc. Psychiatry 41, 811-819. doi: 10. 1097/00004583-200207000-00013

Enriquez-Geppert, S., Huster, R. J., Scharfenort, R., Mokom, Z. N., Zimmermann, J., and Herrmann, C. S. (2014). Modulation of frontal-midline theta by neurofeedback. Biol. Psychol. 95, 59-69. doi: 10.1016/j.biopsycho.2013.02.019

Fan, J., McCandliss, B. D., Sommer, T., Raz, A., and Posner, M. I. (2002). Testing the efficiency and independence of attentional networks. J. Cogn. Neurosci. 14, 340-347. doi: 10.1162/089892902317361886

Gevensleben, H., Holl, B., Albrecht, B., Schlamp, D., Kratz, O., Studer, P., et al. (2009b). Distinct EEG effects related to neurofeedback training in children with ADHD: a randomized controlled trial. Int. J. Psychophysiol. 74, 149-157. doi: 10. 1016/j.ijpsycho.2009.08.005 
Gevensleben, H., Holl, B., Albrecht, B., Vogel, C., Schlamp, D., Kratz, O., et al. (2009a). Is neurofeedback an efficacious treatment for ADHD? A randomised controlled clinical trial. J. Child Psychol. Psychiatry 50, 780-789. doi: 10.1111/j. 1469-7610.2008.02033.x

Gevensleben, H., Kleemeyer, M., Rothenberger, L. G., Studer, P., Flaig-Röhr, A., Moll, G. H., et al. (2014). Neurofeedback in ADHD: further pieces of the puzzle. Brain Topogr. 27, 20-32. doi: 10.1007/s10548-013-0285-y

Hagemann, D., Hewig, J., Seifert, J., Naumann, E., and Bartussek, D. (2005). The latent state-trait structure of resting EEG asymmetry: replication and extension. Psychophysiology 42, 740-752. doi: 10.1111/j.1469-8986.2005. 00367.x

Heinrich, H., Gevensleben, H., and Strehl, U. (2007). Annotation: neurofeedbacktrain your brain to train behaviour. J. Child Psychol. Psychiatry 48, 3-16. doi: 10. $1111 /$ j.1469-7610.2006.01665.x

Jensen, O., and Tesche, C. D. (2002). Frontal theta activity in humans increases with memory load in a working memory task. Eur. J. Neurosci. 15, 1395-1399. doi: 10. 1046/j.1460-9568.2002.01975.x

Jung, T. P., Makeig, S., Westerfield, M., Townsend, J., Courchesne, E., and Sejnowski, T. J. (2000). Removal of eye activity artifacts from visual event-related potentials in normal and clinical subjects. Clin. Neurophysiol. 111, 1745-1758. doi: 10 . 1016/s1388-2457(00)00386-2

Karalunas, S. L., Geurts, H. M., Konrad, K., Bender, S., and Nigg, J. T. (2014), Annual research review: reaction time variability in ADHD and autism spectrum disorders: measurement and mechanisms of a proposed trans-diagnostic phenotype. J. Child Psychol. Psychiatry 55, 685-710. doi: 10.1111/jcpp. 12217

Kofler, M. J., Rapport, M. D., Sarver, D. E., Raiker, J. S., Orban, S. A., Friedman, L. M., et al. (2013). Reaction time variability in ADHD: a meta-analytic review of 319 studies. Clin. Psychol. Rev. 33, 795-811. doi: 10.1016/j.cpr.2013.06.001

Kratz, O., Studer, P., Baack, J., Malcherek, S., Erbe, K., Moll, G. H., et al. (2012). Differential effects of methylphenidate and atomoxetine on attentional processes in children with ADHD: an event-related potential study using the attention network test. Prog. Neuropsychopharmacol. Biol. Psychiatry 37, 81-89. doi: 10. 1016/j.pnpbp.2011.12.008

Kratz, O., Studer, P., Malcherek, S., Erbe, K., Moll, G. H., and Heinrich, H. (2011). Attentional processes in children with ADHD: an event-related potential study using the attention network test. Int. J. Psychophysiol. 81, 82-90. doi: 10.1016/j. ijpsycho.2011.05.008

Lazzaro, I., Gordon, E., Whitmont, S., Meares, R., and Clarke, S. (2001). The modulation of late component event related potentials by pre-stimulus
EEG theta activity in ADHD. Int. J. Neurosci. 107, 247-264. doi: 10. 3109/00207450109150688

Liechti, M. D., Valko, L., Müller, U. C., Döhnert, M., Drechsler, R., Steinhausen, H. C., et al. (2013). Diagnostic value of resting electroencephalogram in attention-deficit/hyperactivity disorder across the lifespan. Brain Topogr. 26, 135-151. doi: 10.1007/s10548-012-0258-6

Loo, S. K., and Smalley, S. L. (2008). Preliminary report of familial clustering of EEG measures in ADHD. Am. J. Med. Genet. B Neuropsychiatr. Genet. 147B, 107109. doi: 10.1002/ajmg.b.30575

Monastra, V. J., Lubar, J. F., Linden, M., VanDeusen, P., Green, G., Wing, W., et al. (1999). Assessing attention deficit hyperactivity disorder via quantitative electroencephalography: an initial validation study. Neuropsychology 13, 424433. doi: 10.1037//0894-4105.13.3.424

Ogrim, G., Kropotov, J., and Hestad, K. (2012). The quantitative EEG theta/beta ratio in attention deficit/hyperactivity disorder and normal controls: sensitivity, specificity and behavioral correlates. Psychiatry Res. 198, 482-488. doi: 10. 1016/j.psychres.2011.12.041

Rueda, M. R., Fan, J., McCandliss, B. D., Halparin, J. D., Gruber, D. B., Lercari, L. P., et al. (2004). Development of attentional networks in childhood. Neuropsychologia 42, 1029-1040. doi: 10.1016/j.neuropsychologia.2003.12.012

Snyder, S. M., Quintana, H., Sexson, S. B., Knott, P., Haque, A. F., and Reynolds, D. A. (2008). Blinded, multi-center validation of EEG and rating scales in identifying ADHD within a clinical sample. Psychiatry Res. 30, 346-538. doi: 10. 1016/j.psychres.2007.05.006

Conflict of Interest Statement: The authors declare that the research was conducted in the absence of any commercial or financial relationships that could be construed as a potential conflict of interest.

Received: 08 May 2014; accepted: 21 July 2014; published online: 21 August 2014. Citation: Heinrich H, Busch K, Studer P, Erbe K, Moll GH and Kratz O (2014) EEG spectral analysis of attention in ADHD: implications for neurofeedback training? Front. Hum. Neurosci. 8:611. doi: 10.3389/fnhum.2014.00611

This article was submitted to the journal Frontiers in Human Neuroscience.

Copyright (c) 2014 Heinrich, Busch, Studer, Erbe, Moll and Kratz. This is an openaccess article distributed under the terms of the Creative Commons Attribution License (CC BY). The use, distribution or reproduction in other forums is permitted, provided the original author(s) or licensor are credited and that the original publication in this journal is cited, in accordance with accepted academic practice. No use, distribution or reproduction is permitted which does not comply with these terms. 